\title{
Avulsão das origens lateral e medial do músculo gastrocnêmio em gata: relato de caso
}

[Avulsion of the lateral and medial heads of the gastrocnemius muscle in a queen: a case report]

\author{
E.A. Tudury ${ }^{1}$, M.L. Figueiredo ${ }^{2}$, C.E.S. Silva ${ }^{3}$, B.M. Araújo ${ }^{2}$, T.H.T. Fernandes ${ }^{2}$, \\ A.C. Silva ${ }^{2}$, M.M.A. Amorim ${ }^{4}$ \\ ${ }^{1}$ Universidade Federal Rural de Pernambuco - Recife, PE \\ ${ }^{2}$ Alunos de pós-graduação - Universidade Federal Rural de Pernambuco - Recife, PE \\ ${ }^{3}$ Residente - Hospital Veterinário - Universidade Federal Rural de Pernambuco - Recife, PE \\ ${ }^{4}$ Aluna de graduação - Universidade Federal Rural de Pernambuco - Recife, PE
}

\begin{abstract}
RESUMO
Poucos relatos têm descrito avulsões das cabeças lateral ou medial e avulsão bilateral das origens do músculo gastrocnêmio em cães e gatos. Este trabalho descreveu o primeiro relato de avulsão das origens lateral e medial do músculo gastrocnêmio em gata, bem como a técnica de reinserção cirúrgica. Uma gata, sem raça definida, com 10 anos, apresentou claudicação do membro posterior direito com postura plantígrada. Avulsão bilateral das cabeças do músculo gastrocnêmio foi diagnosticada com base nos achados do exame clínico. A técnica de reinserção foi realizada com fio mononáilon, numa sutura de Kessler modificada, ancorada em um orifício criado na porção metafisária distal do fêmur. Após período de imobilização externa complementar seguida de fisioterapia, houve recuperação satisfatória.
\end{abstract}

Palavras-chave: gato, tendão de Aquiles, ruptura proximal

\begin{abstract}
Few reports have described avulsions of the medial or lateral heads, as well as bilateral avulsion of the origins of the gastrocnemius muscle in dogs and cats. The objective of this study was to describe the first report of avulsion of the origins of the lateral and medial gastrocnemius muscle in a cat (Felis catus), as well as the surgical reinsertion technique. A mixed breed queen at 10 years of age presented claudication of the right hind limb with plantigrade stance. Bilateral avulsion of the heads of the gastrocnemius muscle was diagnosed based on findings from clinical a examination. The reinsertion technique was performed with monofilament nylon thread in a modified Kessler suture anchored in a hole created in the distal metaphyseal portion of the femur, obtaining satisfactory recovery after a period of additional external immobilization followed by physical therapy.
\end{abstract}

Keywords: cat, Achilles tendon, proximal rupture

\section{INTRODUÇÃO}

O tendão calcâneo comum - tendão de Aquiles é composto por três tendões: tendão do músculo gastrocnêmio, tendão do flexor digital superficial e tendão comum dos músculos bíceps femoral, grácil e semitendinoso (Hermanson e Evans, 1993), sendo o tendão do gastrocnêmio seu principal componente (Dyce et al., 1997). O músculo gastrocnêmio possui duas cabeças inseridas nas tuberosidades supracondilares medial e lateral do fêmur, que incluem o tendão flexor digital superficial entre elas. $\mathrm{Na}$ origem de cada tendão, existe um osso sesamoide (fabela), que articula com o côndilo femoral correspondente. As duas cabeças unem-se na parte proximal da perna e dão origem a um único tendão, que se insere na superfície dorsal proximal do calcâneo (Hermanson e Evans, 1993; Dyce et al., 1997). A ação principal do

Recebido em 5 de novembro de 2011

Aceito em 7 de fevereiro de 2013

*Autor para correspondência (corresponding author)

E-mail: marcellalf@hotmail.com 
músculo gastrocnêmio é a extensão do tarso, mas ele também atua na flexão do joelho (Hermanson e Evans, 1993; Dyce et al., 1997; Stahl et al., 2010).

As lesões ao músculo gastrocnêmio podem ocorrer na origem das cabeças lateral ou medial, no ventre muscular, na junção musculotendínea, no tendão e na região de inserção do tendão no calcâneo. Lesões nas áreas distal e média são mais comuns, enquanto a região proximal é raramente afetada (Muir e Dueland, 1994). A avulsão das origens do músculo gastrocnêmio pode ser parcial ou total (Stahl et al., 2010). Poucos relatos têm descrito avulsões das cabeças lateral (Vaughan, 1979; Reinke et al., 1982; Prior, 1994; Ridge e Owen, 2005) ou medial (Chaffee e Knecht, 1975; Muir e Dueland, 1994), bem como avulsão bilateral da origem do músculo gastrocnêmio em cães (Robinson, 1999). Recentemente foi relatado um caso de avulsão da cabeça lateral em gato (Bali, 2011).

Em geral, a ruptura total está relacionada com trauma agudo, e os animais apresentam claudicação que é imediatamente associada com uma postura plantígrada (Ridge e Owen, 2005). $\mathrm{O}$ diagnóstico frequentemente é feito com base no exame clínico, incluindo a observação da postura e a marcha do animal, bem como a palpação do membro afetado (King e Jerram, 2003; Swiderski et al., 2005). O exame radiográfico com o jarrete flexionado pode revelar deslocamento distal da fabela da cabeça do gastrocnêmio correspondente (Roe, 1998; Ridge e Owen, 2005; Stahl et al., 2010). É importante excluir outras condições, como paralisia do nervo ciático e hiperflexão do tarso congênita, que podem apresentar-se de forma semelhante (King e Jerram, 2003).

Tratamento cirúrgico é preferível ao tratamento médico conservador nos casos de ruptura completa do tendão do gastrocnêmio, uma vez que recidivas são comuns nos casos tratados pelo método conservativo (King e Jerram, 2003). Reinserção cirúrgica ao fêmur, utilizando-se fio monofilamentar resistente ou cerclagem, ancorados ao redor da fabela e em orifícios perfurados no tubérculo supracondilar do fêmur, é geralmente bem-sucedida (Roe, 1998).

Durante o pós-operatório, o membro deve ficar imobilizado, com o jarrete em extensão, por quatro semanas a três meses, para reduzir a tensão sobre o reparo cirúrgico e permitir a cicatrização (Robinson, 1999; King e Jerram, 2003). As técnicas de imobilização incluem fixador esquelético externo transarticular, parafuso trans-calcaneotibial e vários tipos de talas e órteses (Spinella et al., 2010).

Objetivou-se descrever o primeiro relato de avulsão das origens lateral e medial do músculo gastrocnêmio em gata, bem como a técnica de reinserção cirúrgica.

\section{CASUÍSTICA}

Uma gata sem raça definida, com 10 anos de idade e pesando $3,9 \mathrm{~kg}$ foi atendida em Hospital Veterinário Escola por apresentar claudicação do membro pélvico direito com postura plantígrada. A tutora relatou que há uma semana o animal tinha fugido e retornara com quadro de claudicação.

No exame físico, além do posicionamento plantígrado do membro posterior direito, o animal apresentou desconforto à palpação caudal do joelho, onde foi observada uma massa muscular tensa situada no nível da epífise tibial proximal, correspondendo à porção proximal do músculo gastrocnêmio. Nenhuma alteração foi encontrada à palpação do calcâneo e das porções distal e média do tendão calcâneo comum, assim como outros achados ortopédicos. O exame neurológico não revelou disfunções.

Foram solicitadas radiografias em projeções craniocaudal e mediolateral do membro afetado, nas quais não foram encontradas alterações. Com base nos achados do exame físico, foi feito o diagnóstico presuntivo de avulsão bilateral das origens do músculo gastrocnêmio, e o animal foi encaminhado para a reconstituição cirúrgica.

Após as devidas medidas de tricotomia, antissepsia, profilaxia antibiótica e anestesia, o animal foi posicionado em decúbito lateral esquerdo. A seguir, foi feita uma abordagem cranial ao joelho, para ter acesso às cabeças lateral e medial do músculo gastrocnêmio, que se encontravam avulsionadas. Para a reinserção de suas origens, foi feita uma sutura de Kessler modificada, utilizando-se fio mononáilon 2-0. A sutura foi fixada inicialmente na cabeça lateral do músculo gastrocnêmio (Fig. 1A). 
Posteriormente, o fio foi passado caudal à articulação do joelho, em direção medial, para realização de outra fixação na cabeça medial do músculo (Fig. 1B). Em seguida, foi feito um orifício com diâmetro de $1,5 \mathrm{~mm}$, de lateral para medial, na porção distal da metáfise do fêmur, $5 \mathrm{~mm}$ acima das fabelas. A extremidade do fio de sutura emergindo da cabeça lateral foi passada através desse orifício, em direção à face medial do fêmur (Fig. 1C), para se encontrar com a extremidade do fio da cabeça medial, finalizando a sutura (Fig. 1D). Esse procedimento foi realizado novamente com outro fio, no intuito de reforçar a ancoragem. No seu trajeto em sentido proximal, os fios provenientes das cabeças lateral e medial do músculo gastrocnêmio foram passados internamente às inserções dos músculos bíceps femoral (lateral) e grácil e semimembranoso (medial). A sutura dos tecidos foi feita de modo rotineiro, com exceção da pele, onde foi realizada uma sutura intradérmica contínua.

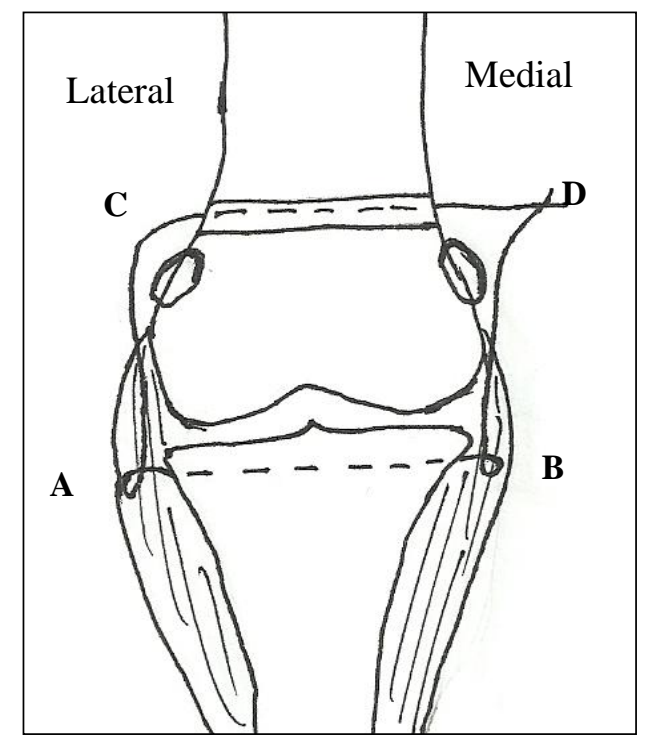

Figura 1. Passo a passo (A, B, C e D) da sutura de Kessler modificada usada para reinserção das cabeças lateral e medial do músculo gastrocnêmio à metáfise femoral distal. Fonte: Figueiredo, 2011.

Uma tala de Robert Jones modificada foi colocada no membro operado, com o jarrete em extensão, por 30 dias. Os cuidados pós-operatórios incluíram anti-inflamatório (meloxicam - Maxicam 0,5mg, Ouro Fino. São
Paulo-SP, 0,1mg/kg, SID, sete dias), analgésico (tramadol - Dorless 12mg, Agener União, $2 \mathrm{mg} / \mathrm{kg}$, TID, quatro dias), antibiótico (cefalexina - Cefalexina Farmácia de Manipulação da Universidade Federal de Pernambuco, 30mg/kg, TID, oito dias) e repouso por 90 dias.

Após 30 dias, o animal retornou para retirada da imobilização e, na avaliação, observou-se que ele caminhava com a articulação tarsocrural rígida e em extensão, apoiando com o dorso dos dígitos (Fig. 2). Foi, então, prescrita fisioterapia passiva do membro. Após uma semana, o animal já tinha um pouco de mobilidade da articulação tibiotársica, mas continuava a pisar com o dorso da pata. Para ajudar na deambulação, foi feita uma bandagem na extremidade da pata, semelhante àquela utilizada para animais com lesão do nervo ciático (Slocum e Slocum, 1998), e implementada fisioterapia com FES (Functional Electrical Stimulation) na musculatura do quadríceps, laser no tendão calcâneo comum e campo magnético em todo o membro, por três semanas. Após esse período, o animal voltou a pisar com os coxins e a deambular normalmente. Aos seis meses de pósoperatório, mantinha boa deambulação.

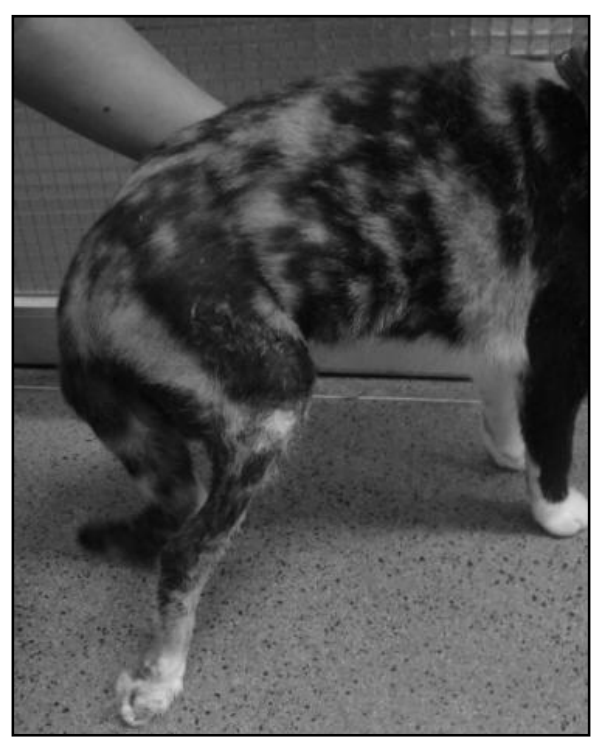

Figura 2. Animal após a retirada da bandagem com 30 dias de pós-operatório. Fonte: Figueiredo, 2011. 


\section{DISCUSSÃO}

Avulsão bilateral das cabeças do músculo gastrocnêmio é raramente relatada como causa de claudicação em cães (Robinson, 1999), o que ainda não foi documentado em gatos. Postura plantígrada bilateral pode surgir em gatos diabéticos devido à polineuropatia distal (Fernández e Bernardini, 2010). Como, nesse caso, a postura plantígrada não acometia os dois membros, suspeitou-se primeiramente de lesão no mecanismo do tendão de Aquiles ou de fratura do calcâneo, que foram rápida e facilmente descartadas à palpação.

De acordo com King e Jerram (2003) e Swiderski et al. (2005), o diagnóstico de avulsão do músculo gastrocnêmio pôde ser feito com base no exame clínico e na observação da marcha. Diferentemente do citado na literatura, o exame radiográfico não revelou deslocamento distal das fabelas, possivelmente porque não foi realizado com o jarrete flexionado, como recomendaram Roe (1998), Ridge e Owen (2005) e Stahl et al. (2010), ou porque as estruturas que fixam as fabelas ainda se encontravam atuantes.

Como recomendado por Robinson (1999) e King e Jerram (2003), o membro do animal foi imobilizado com o jarrete extendido por 30 dias, para impedir a movimentação dessa articulação e, dessa forma, evitar tensão no reparo cirúrgico, permitindo sua cicatrização. De início, pensou-se em imobilizar a articulação tibiotársica com um parafuso ou pino transcalcaneotibial, mas, diante da reduzida espessura do osso tarsal, não foi possível. O membro foi, então, imobilizado com uma tala de Robert Jones modificada, o que também é indicado por Spinella et al. (2010), tendo, neste caso, resultado satisfatório.

Em razão dos 30 dias de imobilização, após a retirada da tala, o animal apresentou o membro rígido, retesamento do tendão de Aquiles e apoio do membro com o dorso dos dedos. Segundo Haapala et al. (1999), imobilização por mais de quatro semanas pode resultar em efeitos deletérios e permanentes para as articulações, bem como atrofia e degeneração dos músculos e tendões (King e Jerram, 2003). Contudo, Virchenko e Aspenberg (2006) preconizam que a imobilização precoce da articulação tibiotársica em lesões do mecanismo do tendão de Aquiles melhora o processo de cicatrização e aumenta a resistência à tração do reparo do tendão. Esse quadro foi corrigido com a implementação da fisioterapia (Spinella et al., 2010) e a bandagem do membro utilizada para lesão do nervo ciático (Slocum e Slocum, 1998), que eleva a extremidade dos dígitos e, assim, impede o apoio incorreto da pata.

Muitos autores utilizam cerclagem ao redor da fabela ao invés de náilon para a reinserção da origem do músculo gastrocnêmio através de um túnel realizado na porção distal do fêmur, podendo-se obter resultado desfavorável em razão da quebra do implante (Robinson, 1999; Ridge e Owen, 2005). Devido à possibilidade dessa intercorrência e frente às propriedades elástica e de resistência do náilon e ao tamanho do paciente, foi utilizado polímero de poliamida monofilamentar com padrão de sutura Kessler modificada, tendo-se, assim, um resultado cirúrgico apropriado e satisfatório.

\section{CONCLUSÕES}

A avulsão de ambas as cabeças, lateral e medial, do músculo gastrocnêmio gera em gatos postura plantígrada e massa palpável e dolorosa caudalmente à articulação do joelho, podendo ser corrigida com fixação dos cotos musculares à metáfise femoral distal, por meio da sutura de Kessler modificada aqui utilizada, junto à manutenção do membro com imobilização externa para proteger o reparo cirúrgico.

\section{REFERÊNCIAS}

BALI, M.S. Avulsion of the lateral head of the gastrocnemius muscle in a cat. J. Feline Med. Sug. v.13, p.784-786, 2011.

CHAFFEE, V.W.; KNECHT, C.D. Avulsion of the medial head of the gastrocnemius in the dog. Vet. Med. Small Anim. Clin., v.70, p.929-931, 1975.

DYCE, K.M.; SACK, W.O.; WENSING, C.J.G. $\mathrm{O}$ aparelho locomotor. In:__. Tratado de anatomia veterinária, 2.ed. Rio de Janeiro: Guanabara Koogan, 1997. p.25-78.

FERNÁNDEZ, V.L.; BERNARDINI, M. Enfermidades metabólicas. In:_. Neurologia em cães e gatos, 1.ed. São Paulo: Med Vet, 2010. p.239-260. 
HAAPALA, J.; AROKOSKI, J.P.; HYTTINEN, M.M. et al. Remobilization does not fully restore immobilization induced articular cartilage atrophy. Clin. Orthop. Relat. Res. V.362, p.218229, 1999.

HERMANSON, J.W.; EVANS, H.E. The muscular system: muscles of the pelvic limb. In: EVANS, H.E. Miller's anatomy of the dog, 3.ed. Philadelphia:W.B Saunders, 1993. p.349-384.

KING, M.; JERRAM, R. Achilles tendon rupture in dogs. Compendium, v.25, p.613-620, 2003.

MUIR, P.; DUELAND, R.T. Avulsion of the origin of the medial head of the gastrocnemius muscle in a dog. Vet. Rec., v.135, p.359-360, 1994.

PRIOR, J.E. Avulsion of the lateral head of the gastrocnemius muscle in a working dog. Vet. Rec., v.134, p.382-383, 1994.

REINKE, J.D.; KUS, S.P.; OWENS, J.M. Traumatic avulsion of the lateral head of the gastrocnemius and superficial digital flexor muscle in a dog. J. Am. Anim. Hosp. Assoc., v.25, p.213-216, 1982.

RIDGE, P.A.; OWEN, M.R. Unusual presentation of avulsion of the lateral head of the gastrocnemius muscle in a dog. J. Small Anim. Pract., v.46, p.196-198, 2005.

ROBINSON, A. Atraumatic bilateral avulsion of the origins of the gastrocnemius muscle. J. Small Anim, Pract., v.40, p.498-500, 1999.
ROE, S.C. Injury na diseases of tendons. In: BLOOMBERG, M.S.; DEE, J.P.; TAYLOR, R.A. Canine Sports Medicine and Surgery, 13.ed. Philadelphia:W.B Saunders, 1998. p.9299.

SLOCUM, B.; SLOCUM, T.D. Bones and joints: bandages for sciatic nerve deficit. In:_. Current techniques in small animal surgery, 4.ed. Baltimore: Willians and Wilkins, 1998. p.13161318.

SPINELLA, G.; TAMBURRO, R.; LOPRETE, G. et al. Surgical repair of achilles tendon rupture in dogs: a review of the literature, a case report and new perspectives. Vet. Med., v.55, p.303-310, 2010.

STAHL, C.; WACKER, C.; WEBER, U. et al. MRI features of gastrocnemius musculotendinopathy in herding dogs. Vet. Radiol. Ultrasound., v.51, p.380-385, 2010.

SWIDERSKI, J.; FITCH, A.; STAATZ, A. et al. Sonographic assisted diagnosis and treatment of bilateral gastrocnemius tendon rupture in a Labrador retriever repaired with fascia lata and polypropylene mesh. Vet Com. Orthop. Traumatol., v.18, p.258-263, 2005.

VAUGHAN, L.C. Muscle and tendon injuries in dogs. J. Small Anim. Pract., v.20, p.711-736, 1979.

VIRCHENKO, O.; ASPENBERG, P. How can one platelet injection after tendon injury lead to a stronger tendon after 4 weeks? Acta Orthop., v.77, p.806-812, 2006. 